Superconducting Super Collider Laboratory

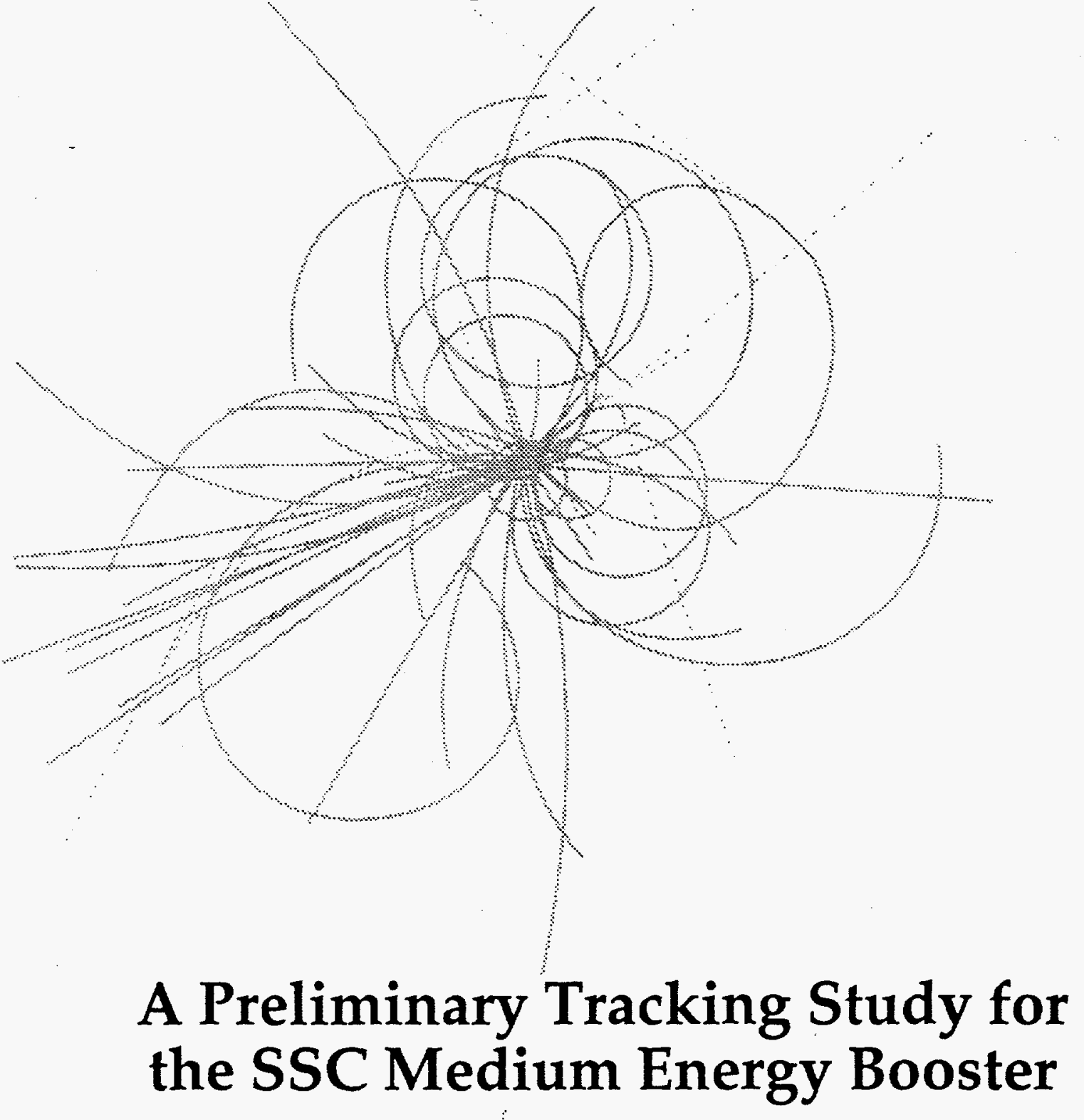

Cleon Manz

January 1990

APPROVED FOR REIEASE OR PUBLICATION - OR. PATENT GROUP BY... E ....... DATE. $1 / 3 / \%$... 
SSCL-298

\title{
A Preliminary Tracking Study for the SSC Medium Energy Booster
}

\author{
Cleon Manz \\ Superconducting Super Collider Laboratory* \\ Accelerator Division \\ 2550 Beckleymeade Avenue \\ Dallas, TX 75237
}

January 1990

\section{DISCLAIMER}

This report was prepared as an account of work sponsored by an agency of the United States Government. Neither the United States Government nor any agency thereof, nor any of their employees, makes any warranty, express or implied, or assumes any legal liability or responsibility for the accuracy, completeness, or usefulness of any information, apparatus, product, or process disclosed, or represents that its use would not infringe privately owned rights. Reference herein to any specific commercial product, process, or service by trade name, trademark, manufacturer, or otherwise does not necessarily constitute or imply its endorsement, recommendation, or favoring by the United States Government or any agency thereof. The views and opinions of authors expressed herein do not necessarily state or reflect those of the United States Government or any agency thereof.

* Operated by the Universities Research Association, for the U.S. Department of Energy under Contract No. DE-AC02-89ER40486. 


\section{DISCLAIMER}

Portions of this document may be illegible in electronic image products. Images are produced from the best available original document. 


\title{
A Preliminary Tracking Study for the SSC Medium Energy Booster
}

\author{
Cleon Manz \\ Superconducting Super Collider Laboratory* \\ Accelerator Division \\ 2550 Beckleymeade Avenue \\ Dallas, TX 75237
}

January 1990

The following is a summary of a preliminary tracking study done on the Medium Energy Booster. The tracking code used was one written by the author specifically for this study. What was needed was an investigation of how the sextupole errors found in the main dipole magnets affected particle motion in the MEB. The dipoles are the same magnets proposed for the Main Injector ring at Fermilab, so it was known that these magnets had a particularly strong sextupole component at high magnet excitation currents.

* Operated by the Universities Research Association, for the U.S. Department of Energy under Contract No. DE-AC02-89ER40486. 


\section{MEBTRACK CODE}

The beamline used in the calculations was a linear FODO lattice with sextupole kicks. The size of the kicks depended on whether the particle was at injection or at high energy. At injection, the beam is larger, but the magnet field quality is better than at high energy, because of magnet saturation at a high field. The natural chromaticity of the machine at both injection and at high energy was corrected by placing sextupole magnets before the quadrupoles. With the addition of the sextupole error at high energy, the strengths of the sextupole magnets increased to correct the chromaticity caused by the multipole. Table 1 lists the values of chromaticity and sextupole strengths present at injection and high energy.

Table 1. Chromaticities and Sextupole Strengths

\begin{tabular}{lccccc}
\hline \hline & $\xi_{x}$ & $\xi_{y}$ & $K_{f}\left(\mathrm{~m}^{-3}\right)$ & $K_{d}\left(\mathrm{~m}^{-3}\right)$ & $K_{b}\left(\mathrm{~m}^{-3}\right)$ \\
\hline Injection & 18.0 & -18.0 & 0.0416693 & 0.0626249 & 0.0 \\
High energy & 142.055 & -132.153 & 0.1847844 & -0.117404 & -0.0066704 \\
\hline \hline
\end{tabular}

The sextupole kick is given by:

$$
\begin{aligned}
& \Delta X^{\prime}=-B_{y} L_{s} /(B \rho)=-K_{f} L_{s} / 2\left(x^{2}-y^{2}\right) \\
& \Delta Y^{\prime}=B_{x} L_{s} /(B \rho)=K_{d} L_{s}(x y)
\end{aligned}
$$

where $K_{f}=B_{f}^{\prime \prime} /(B \rho)$;

$$
K_{d}=B_{d}^{\prime \prime} /(B \rho) ;
$$

$L_{s}=$ sextupole magnet length $(\mathrm{m})$.

The particle was tracked at injection for 45000 turns (corresponding to $0.6 \mathrm{sec}$ for the ring to fill); and for 16000 turns at $200 \mathrm{GeV}$ (corresponding to $0.2 \mathrm{sec}$ 
of circulation). The tune, which was used to calculate the focal length of the quadrupoles, was $\nu_{x, y}=16.6012345$. There is a variation of the program that allows one to use decoupled tunes. This option did not seem to make a considerable amount of difference to the results.

\section{TRACKING RESULTS}

To discover the dynamic aperture, two steps were taken. The first was to determine whether the dynamic aperture existed inside or outside of the beam pipe. This consisted of comparing the tracked $x$ and $y$ coordinates to the pipe parameters $(x=80 \mathrm{~mm}, y=50 \mathrm{~mm})$. The initial starting points $X_{o}$ and $Y_{o}$ were calculated from:

$$
\begin{aligned}
& \hat{X}=X_{o} \sqrt{1+\alpha_{x}^{2}} \\
& \hat{Y}=Y_{o} \sqrt{1+\alpha_{y}^{2}},
\end{aligned}
$$

where $\hat{X}=\operatorname{maximum} X$ displacement $(\mathrm{m})$;

$$
\hat{Y}=\text { maximum } Y \text { displacement }(\mathrm{m}) ;
$$$$
\alpha_{x}=-1 / 2 \beta_{x}^{\prime}=1.73
$$$$
\alpha_{y}=-1 / 2 \beta_{y}^{\prime}=-0.654 \text {. }
$$

The maximum $X$ and $Y$ were chosen to be equal and stepped up until the particle hit the pipe walls or its amplitude became extreme. If the particle reached the pipe limits, the program stopped and listed the coordinates and turns of the lost particle. The phase space ellipse of the particle steadily grew due to the nonlinear coupling of the sextupole fields until it reached the physical limit of the beam pipe. This showed the field quality to be quite good, for it was not the instability of the particle that caused it to be lost but the reaching of the pipe wall. The maximum $x$ and $y$ allowing the beam to complete all turns was $15 \mathrm{~mm}$ at both injection and at high energy (Figures 1 and 2). Figures 3 and 4 show the plots of the particle motion $(x, y$ plot) inside the beam pipe at both injection and $200 \mathrm{GeV}$. 


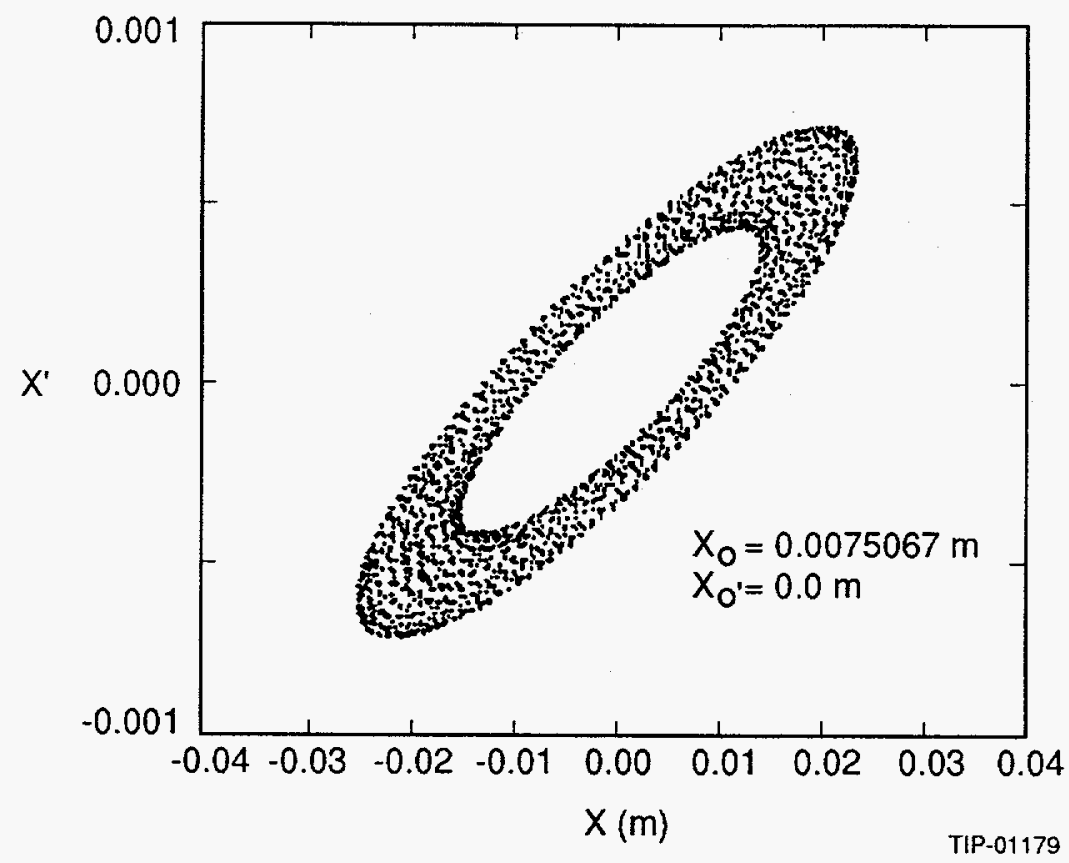

Injection: Horizontal Phase Space, Inside Beam Pipe. $X_{\max }=0.015 \mathrm{~m}$

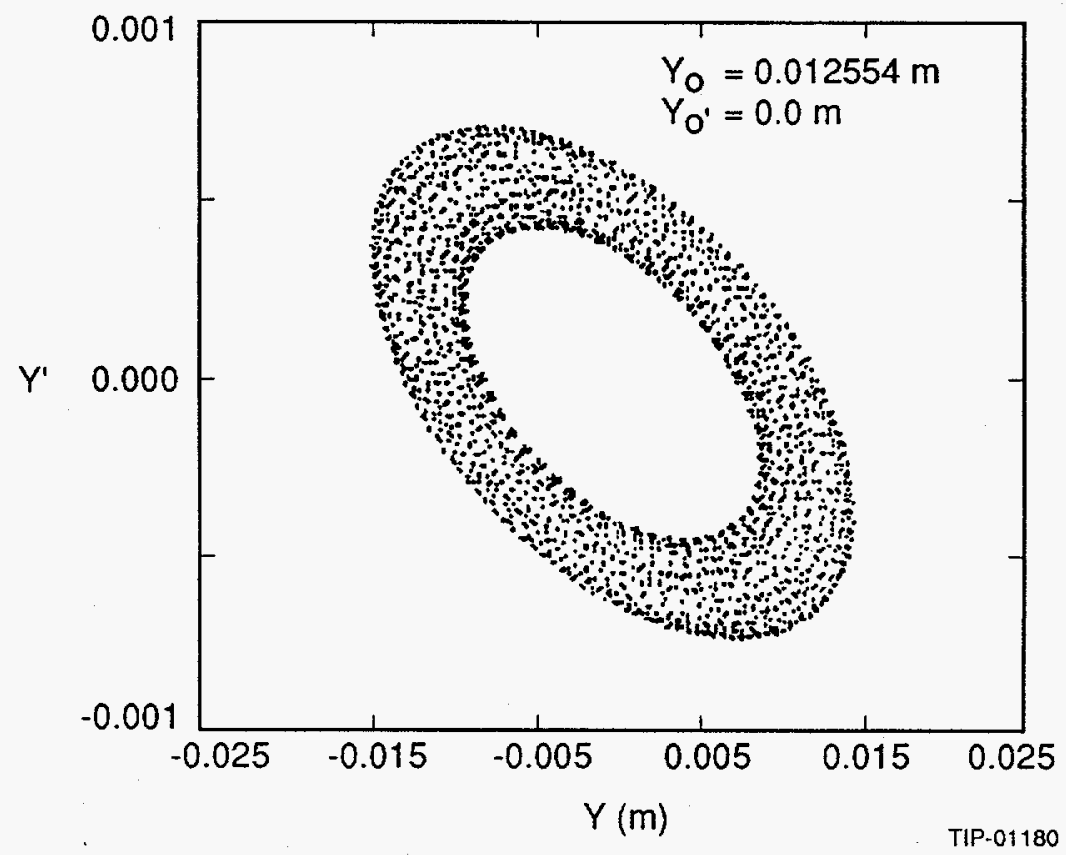

Injection: Vertical Phase Space, Inside Beam Pipe. $Y_{\max }=0.015 \mathrm{~m}$

Figure 1 


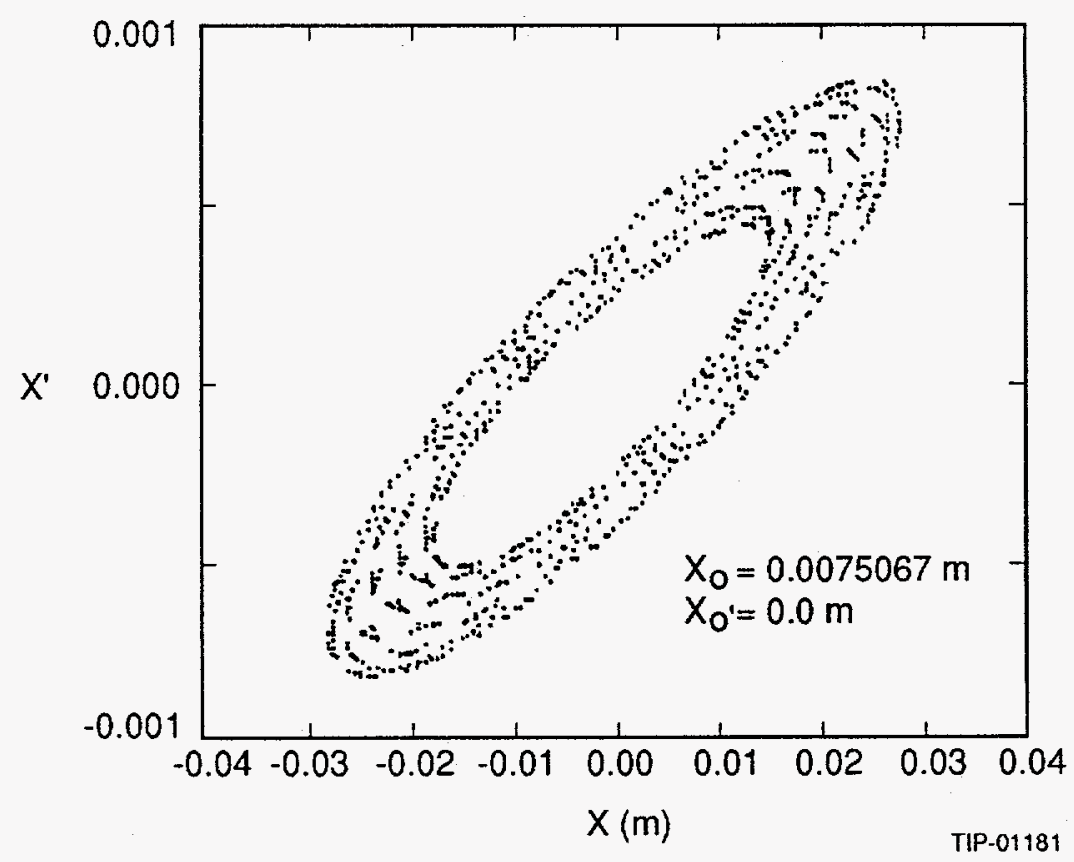

$200 \mathrm{GeV}$ : Horizontal Phase Space, Inside Beam Pipe. $X_{\max }=0.015 \mathrm{~m}$

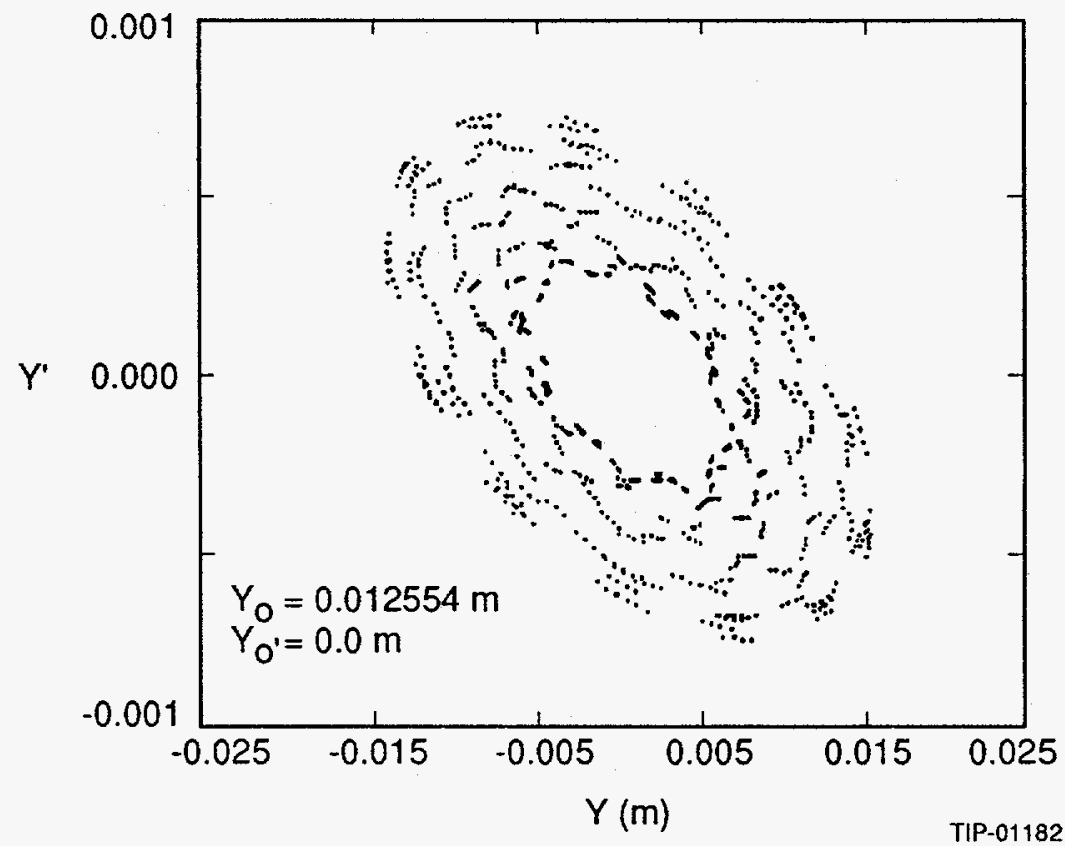

$200 \mathrm{GeV}$ : Vertical Phase Space, Inside Beam Pipe. $Y_{\max }=0.015 \mathrm{~m}$

Figure 2 


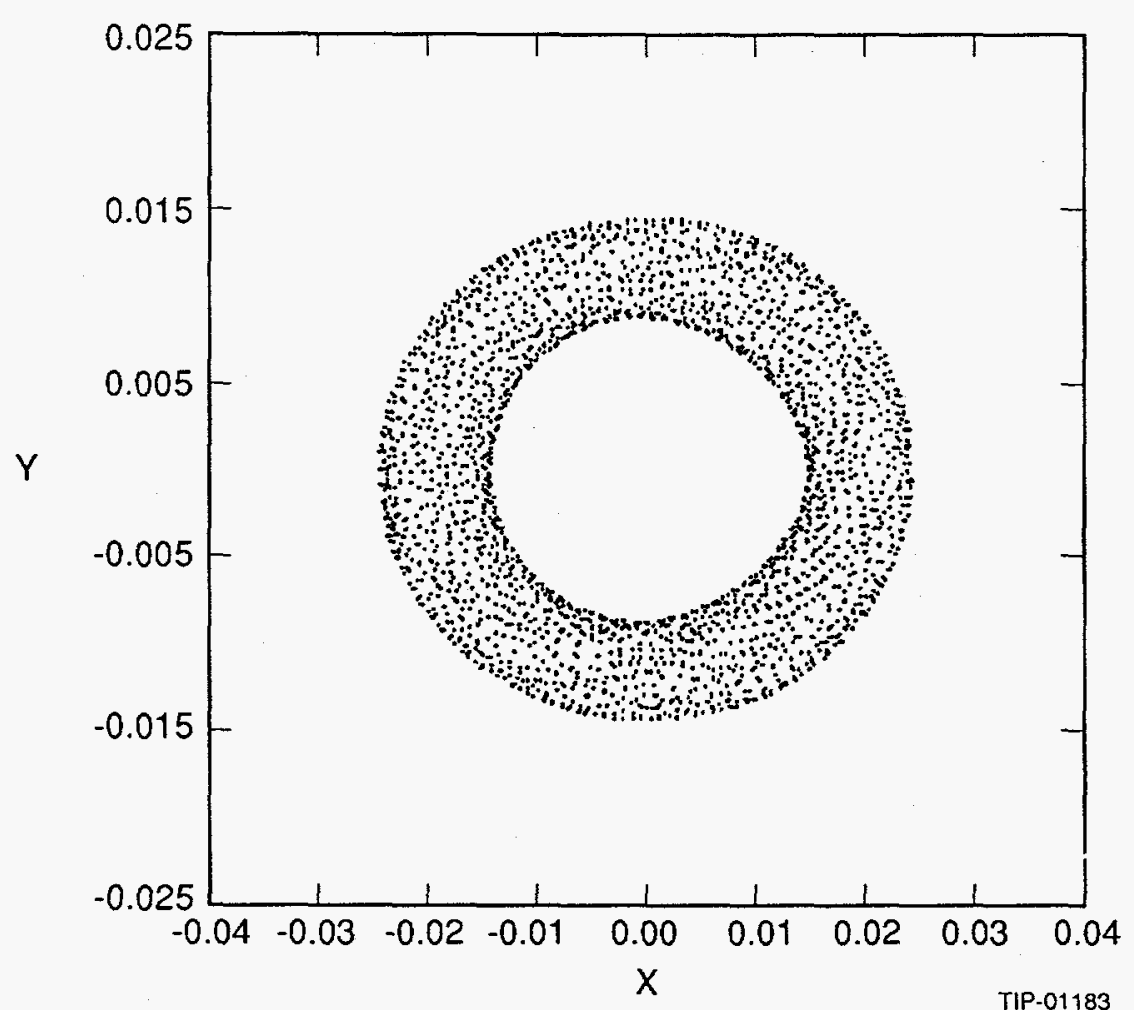

Figure $3 \quad X$ and $Y$ Plot. Beam Pipe

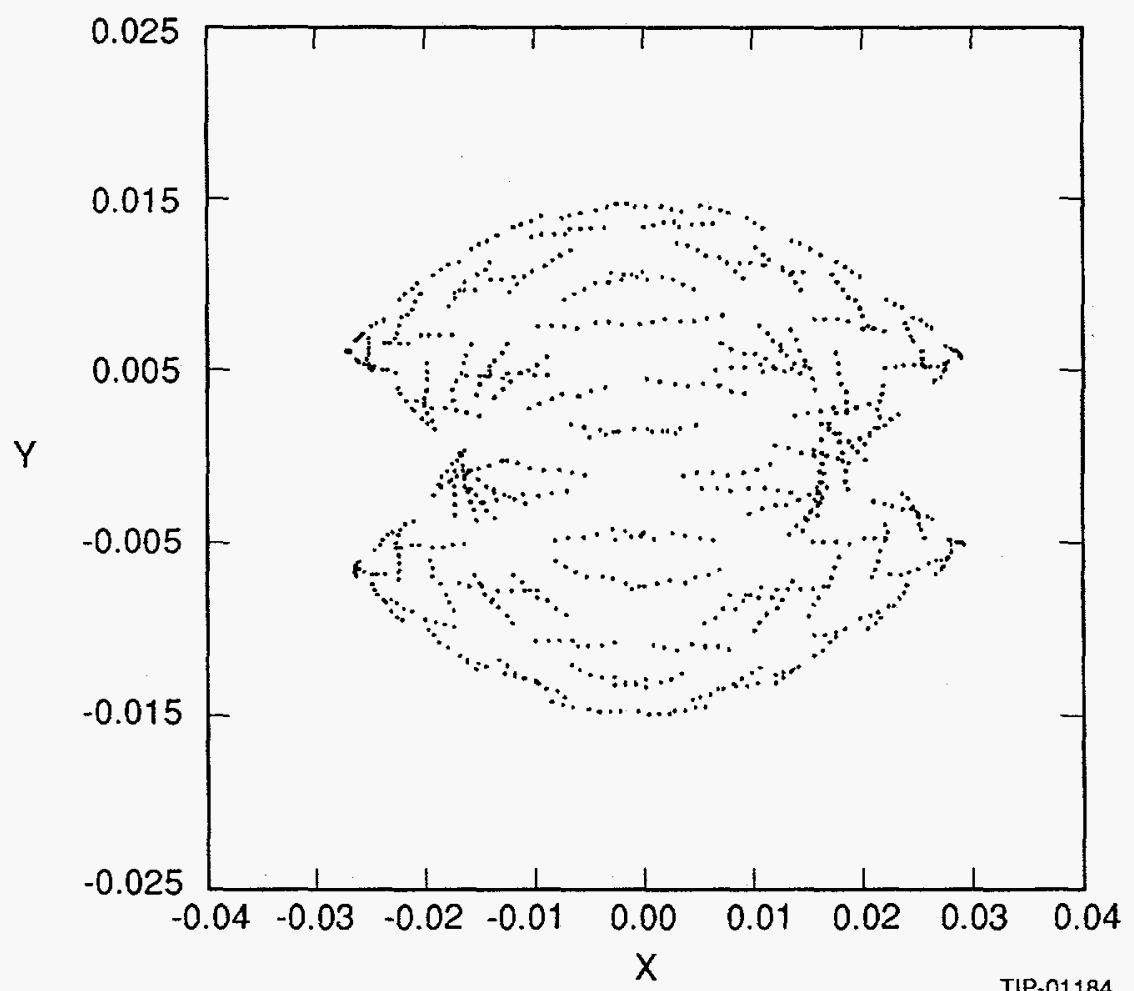

Figure $4200 \mathrm{GeV}$ : Beam Inside Beam Pipe 
The second step involved removing the physical beam pipe, since the dynamic aperture was found to be outside of the pipe. The pipe was made very large $(x=y=20000 \mathrm{~m})$ so that the beam would be lost due to dynamical processes only. What was found was that the dynamic aperture of the beam at injection was $0.295 \mathrm{~m}$; and at high energy it was $0.0805 \mathrm{~m}$. The size of the beam pipe is $0.080 \mathrm{~m}$ horizontal and $0.050 \mathrm{~m}$ vertical (Figures 5 and 6). The dynamic aperture is a very definite point. Figures 7 and 8 show particle motion at a point just beyond the dynamic aperture of the particle. The particle amplitude slowly grows due to the sextupole fields until it receives very massive kicks that cause the amplitude to expand rapidly (Figure 9)! The dynamic aperture of $0.295 \mathrm{~m}$ corresponds to a normalized emittance of $12595.5 \pi \mathrm{mm}$-mrads, calculated from:

$$
\epsilon_{n}=\pi \gamma \hat{X}^{2} / \beta_{x}
$$

where $\gamma=E / E_{o}$;

$\beta_{x}=$ horizontal beta function $(\mathrm{m})$. This is 15000 times the nominal emittance of $0.8 \pi \mathrm{mm}$-mrads. At $200 \mathrm{GeV}$, the aperture of $0.0805 \mathrm{~m}$ corresponds to $20841.6 \pi \mathrm{mm}$-mrads, which is about 26000 times the nominal emittance.

Further studies should be carried out on the tracking of the MEB. This should include more in-depth work on the decoupled tune machine. A version of the tracking code used here does exist with the decoupled tune option. Also, more nonlinearities, such as decapoles, octupoles, etc., could be added to the tracking subroutines in order for the tracking to be more complete. This study consisted of tracking one particle over many turns. The option of adding more than one particle to make a "beam" tracking study could be implemented. 


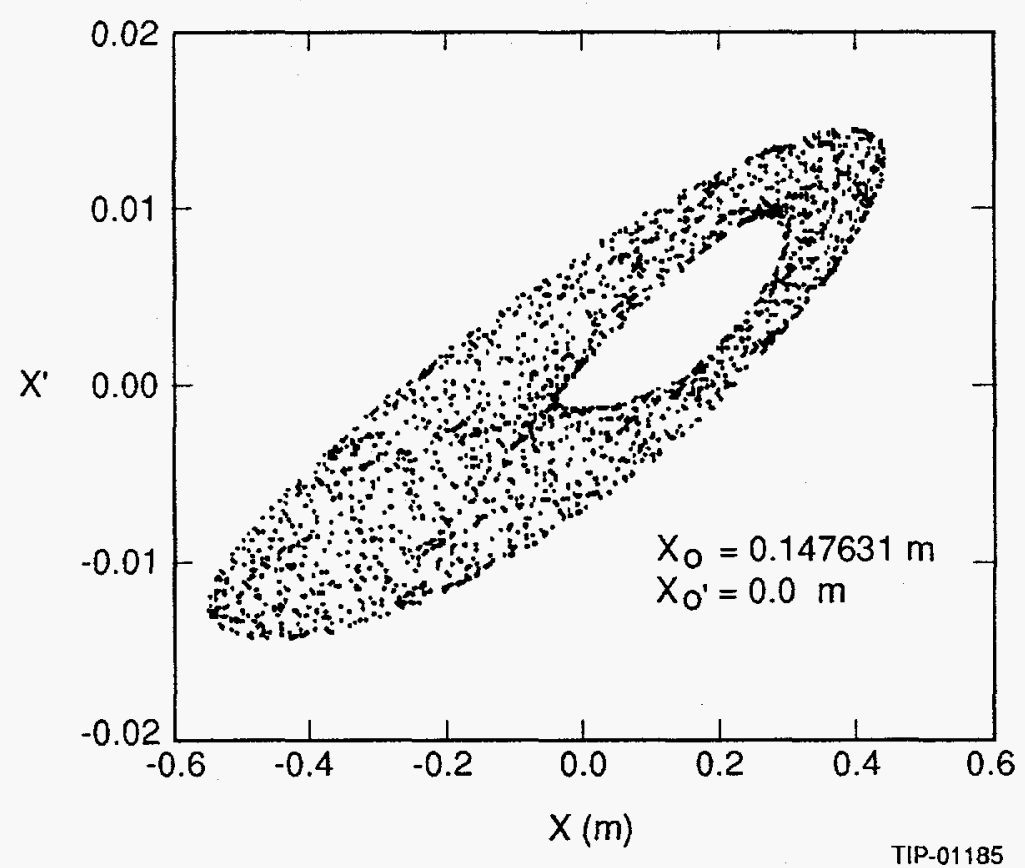

Injection: Dynamic Aperture $=0.295 \mathrm{~m}$, Horizontal Phase Space

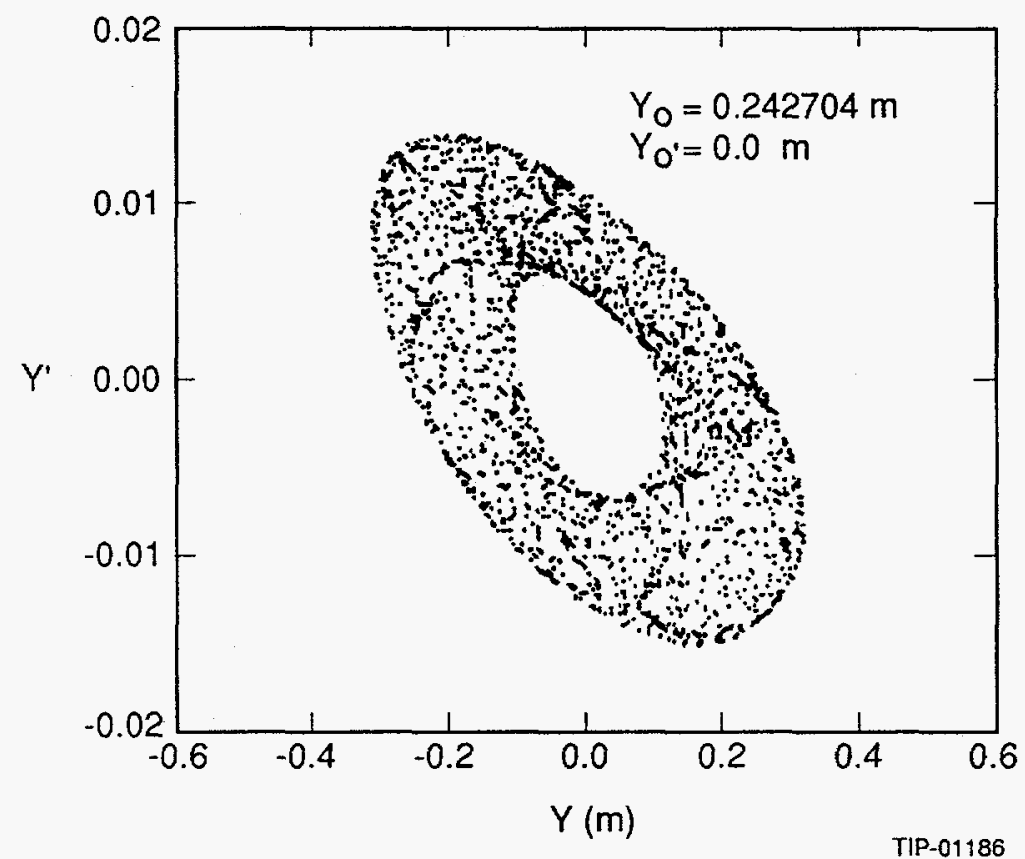

Injection: Dynamic Aperture $=0.295 \mathrm{~m}$, Vertical Phase Space

Figure 5 


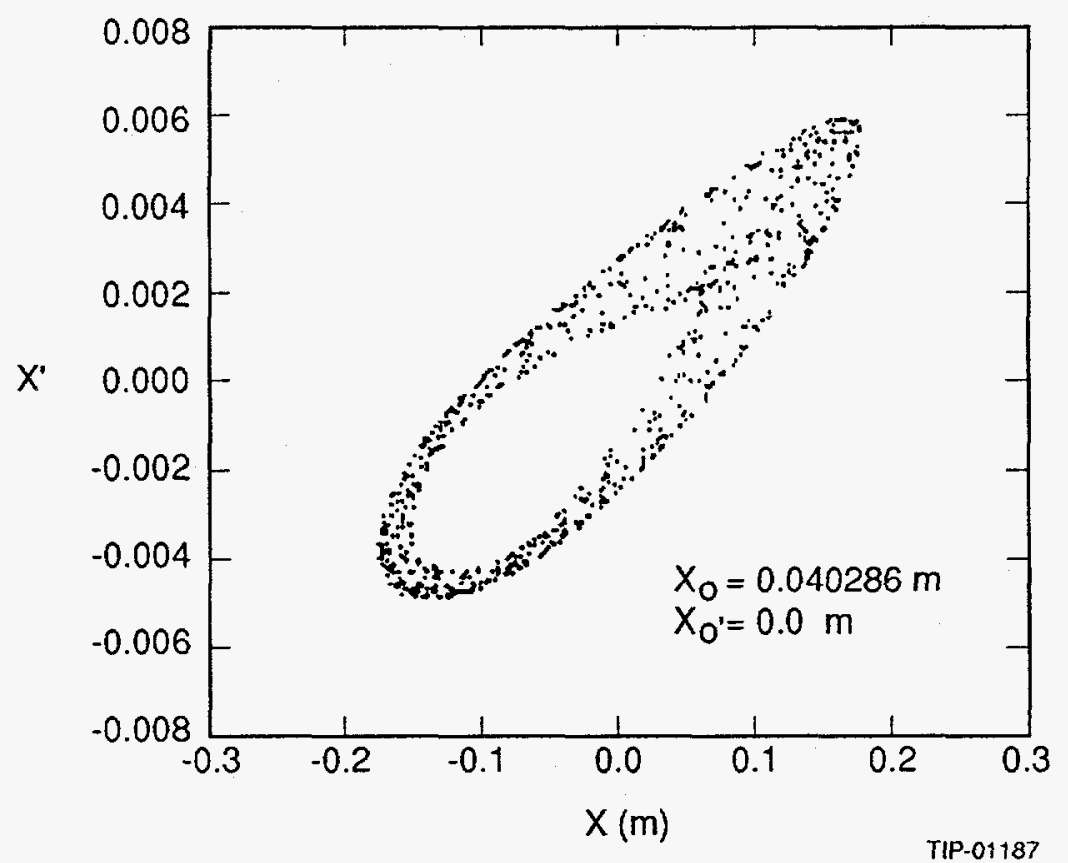

$200 \mathrm{GeV}$ : Dynamic Aperture $=0.0805 \mathrm{~m}$, Horizontal Phase Space

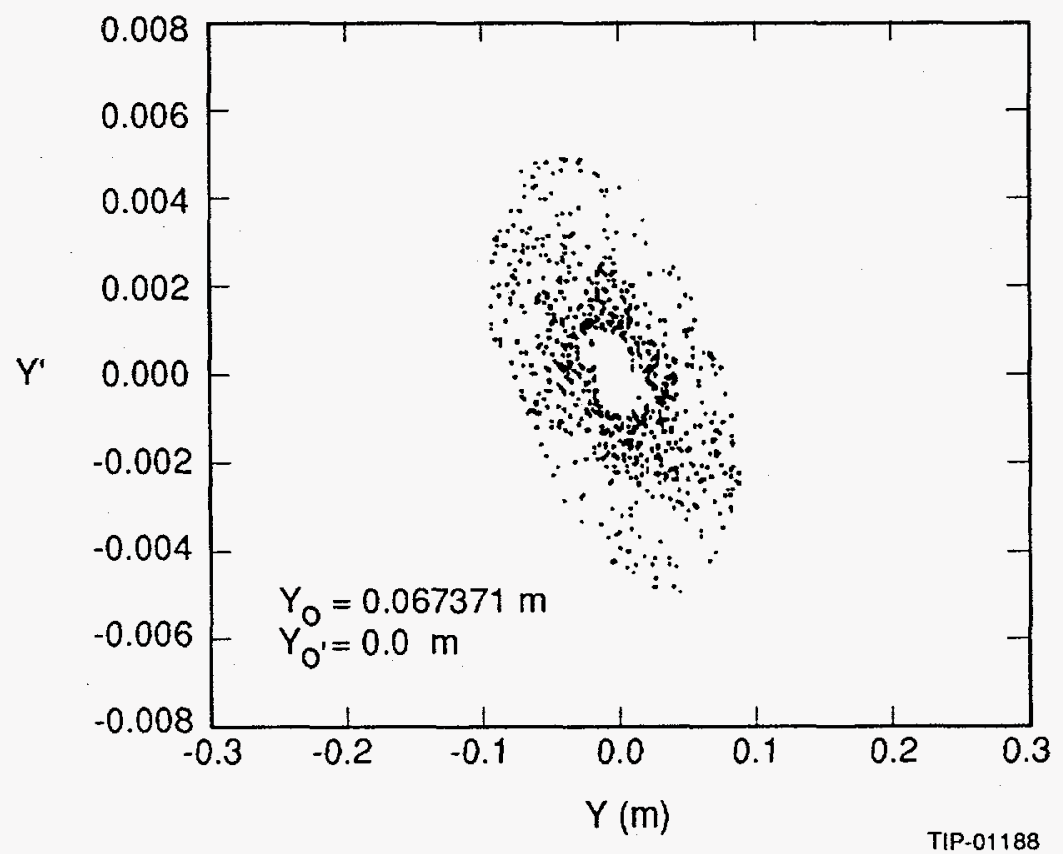

$200 \mathrm{GeV}$ : Dynamic Aperture $=0.0805 \mathrm{~m}$, Vertical Phase Space

Figure 6 
L ว.กริเ

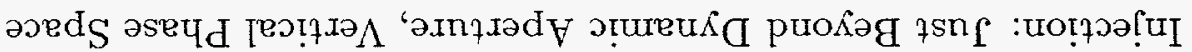

06! 10.dII

(w) $ᄉ$

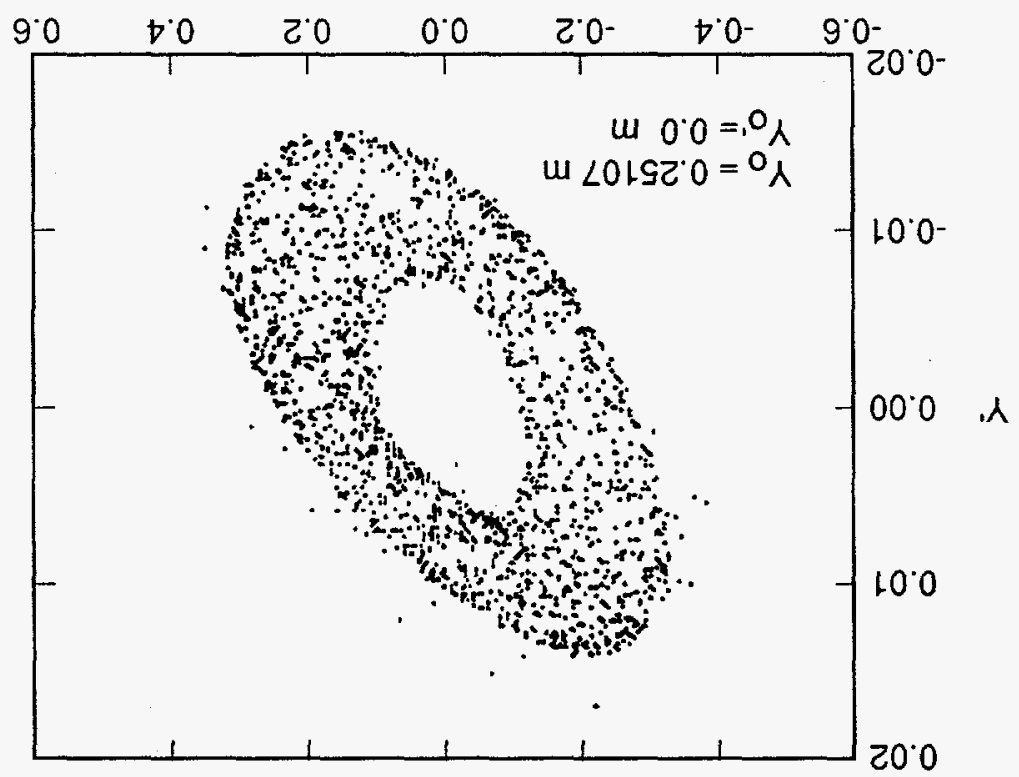

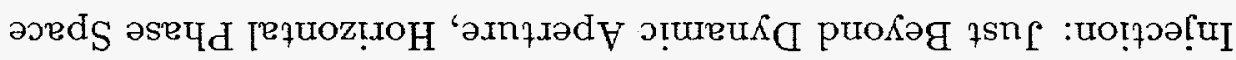

68เ เ0-dII

(w) $x$

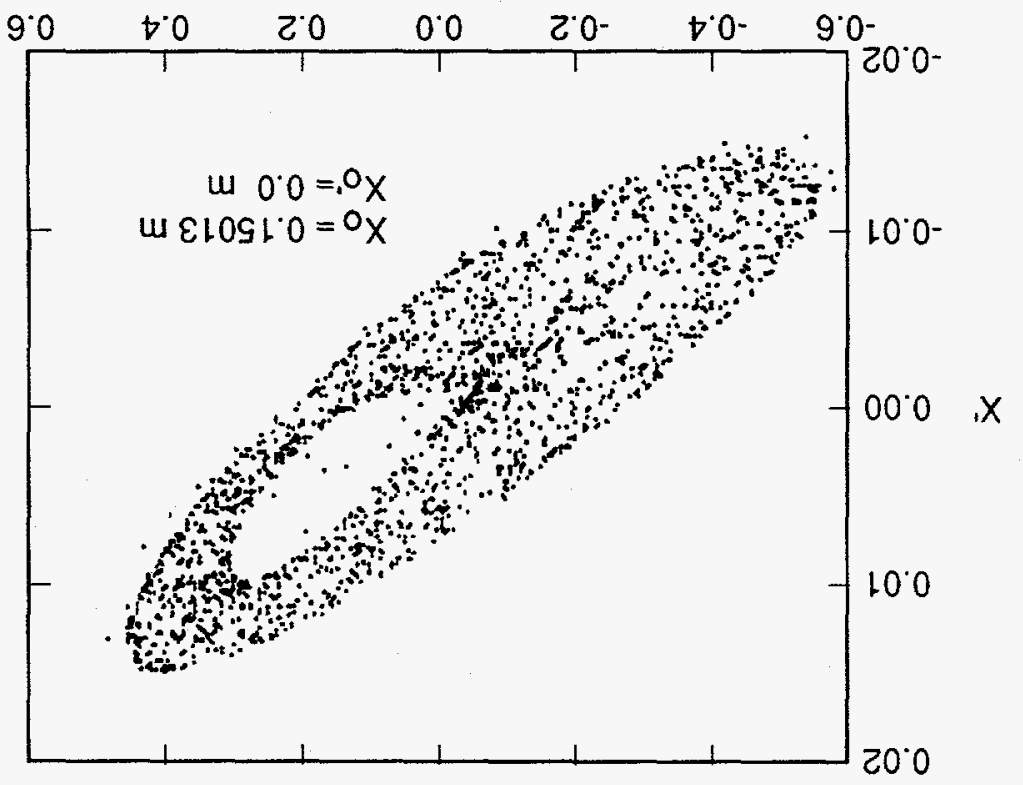




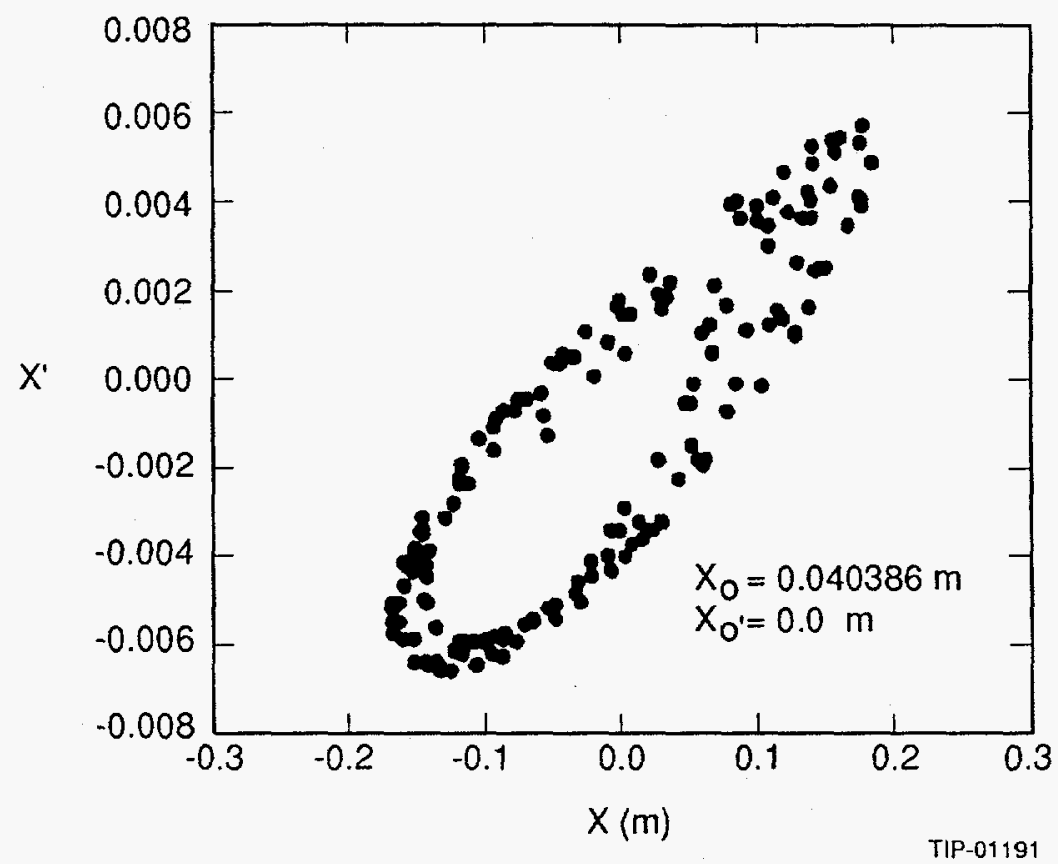

$200 \mathrm{GeV}$ : Just Beyond Dynamic Aperture, Horizontal Phase Space

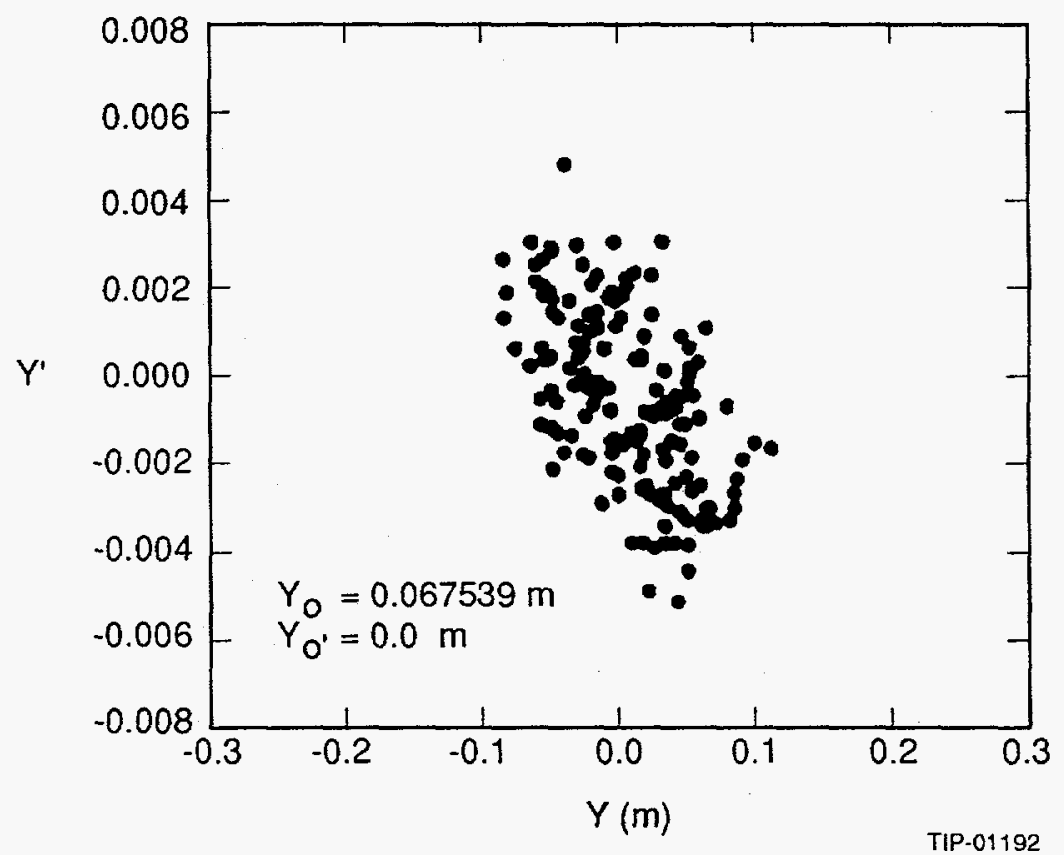

$200 \mathrm{GeV}$ : Just Beyond Dynamic Aperture, Vertical Phase Space

Figure 8 


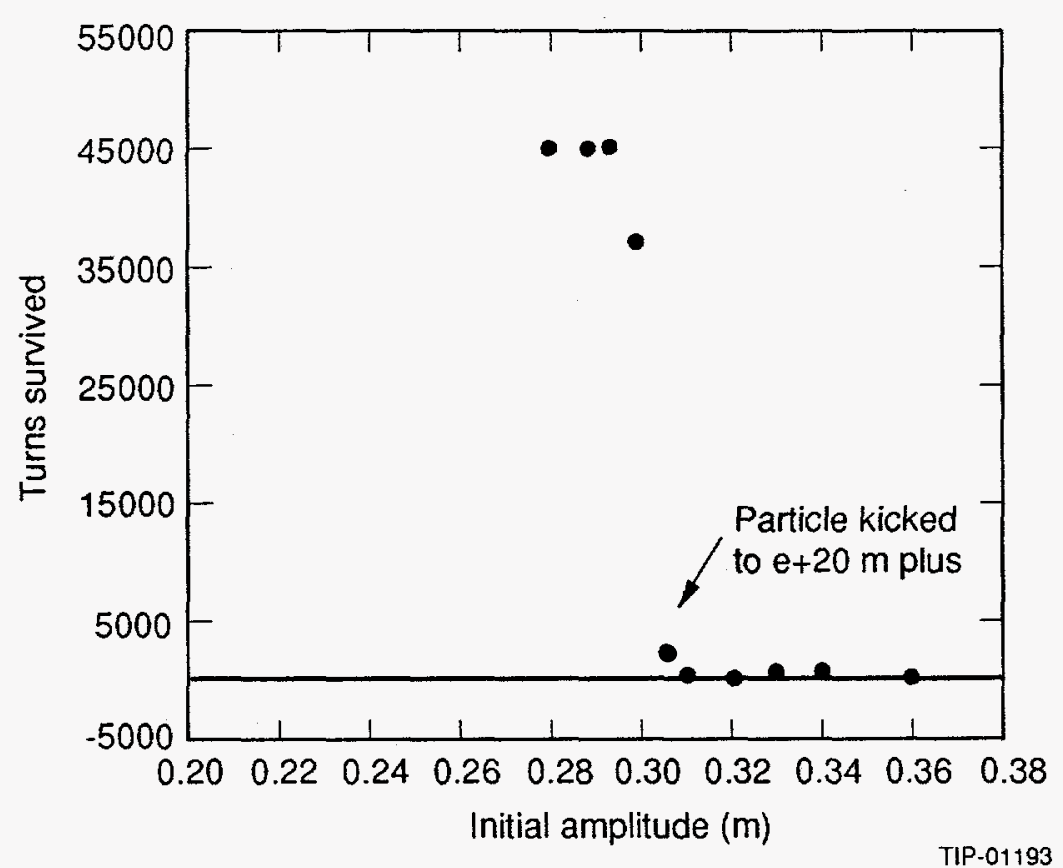

Injection: Dynamic Aperture $=0.295 \mathrm{~m}$

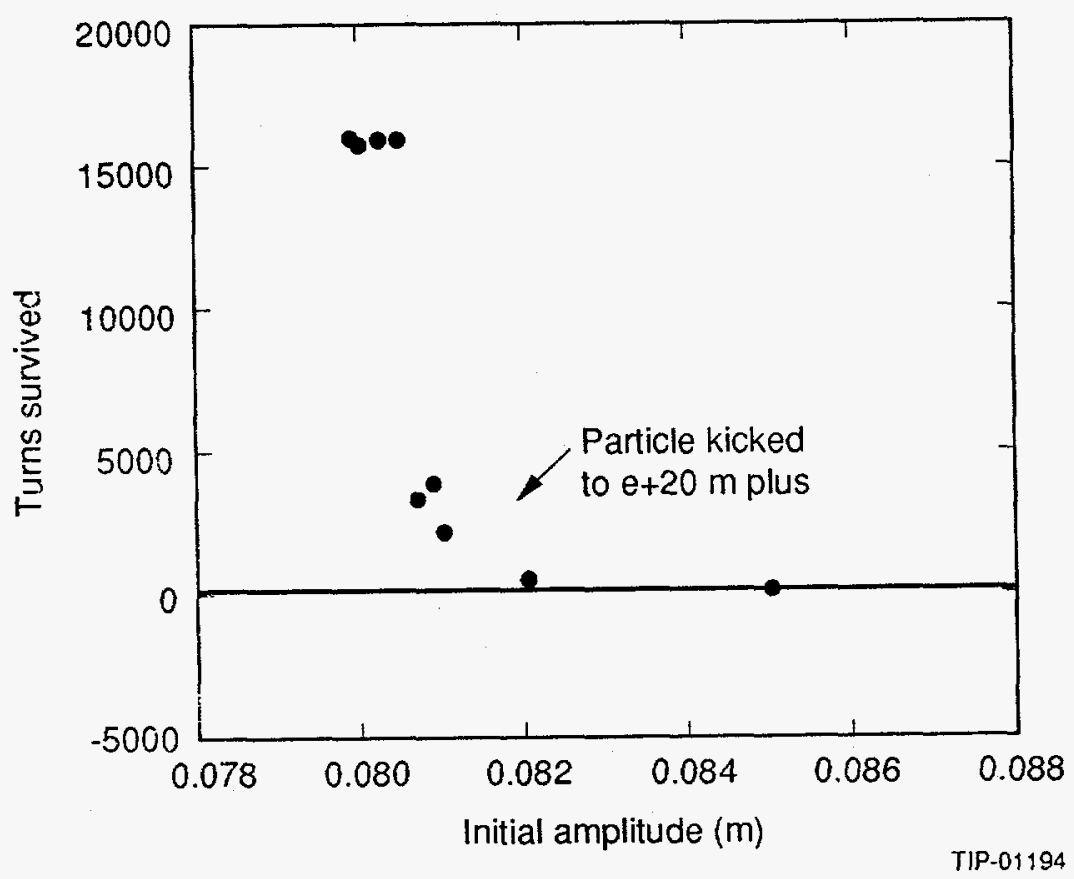

$200 \mathrm{GeV}$ : Dynamic Aperture $=0.0805 \mathrm{~m}$

Figure 9 